\title{
CFD modeling for thermal performance of Closed Loop Pulsating heat pipe in bottom heated mode
}

\author{
Ashutosh Gupta, Ajit K Parwani
}

\begin{abstract}
In this research work a computational model for closed loop pulsating heat pipe (CLPHP) has been developed in order to study its two phase flow process and predict its thermal performance. The pulsating heat pipe of $1.3 \mathrm{~mm}$ internal diameter in vertical bottom heat mode was simulated with unsteady model. Water was taken as working fluid. Volume of fluid model (VOF) in FLUENT was selected for multiphase modelling, also continuum surface force (CSF) model was considered for effects of surface tension. The simulation with unsteady model was successful in reproducing the two phase flow process in CLPHP and oscillation caused by the pressure difference along with the startup characteristics. During the working of CLPHP, lowest thermal resistance of $0.295 \mathrm{~K} / \mathrm{W}$ was achieved. The simulation result obtained is consistent with the experimental results at the same condition.
\end{abstract}

Keywords-Closed Loop Pulsating heat pipe, Computational modelling, bottom heat mode, unsteady model, two phase flow

\section{Introduction}

In order to maintain operational stability of electrical and electronics components, heat or thermal energy must be efficiently transferred to the surrounding. A heat pipe is working on a two phase system has better heat transport capability than a conventional single phase heat exchanger. However, it has various limitations [1], e.g. capillary limit, boiling limit, entrainment limit, sonic limit and viscous limit. Some of these limitations will be overcome by Pulsating heat pipe (PHP) which was introduced by Akachi [2] in the middle of 1990s.

PHP has many advantages over conventional heat pipe, for example it doesn't have wick structure, its miniaturization can be easily made, easy to manufacture and better heat transfer ability. Due to its advantages it has been widely studied and applied in many areas such as thermal management of electronic component, hybrid vehicle, fuel cell cooling, integral thermal spreader, and wire-on-tube heat exchanger [3-6].

The PHP consists of tubes/channels arranged in a serpentine manner and joined end to end. The diameter of the PHP tubes is of capillary diameter so that vapor plugs can be formed due to capillary action. If the diameter is too large, the liquid and vapor phases will stratify. The PHP can operate successfully for all operation modes [6]. Generally, there are two types of PHPs: the looped PHP, which has two open ends, connected to one another, and the unlooped PHP, which has two unconnected ends. Oscillating motions of liquid plugs and vapor slugs in capillary diameter tube, drive the working fluid flowing in the pipe. The liquid plugs and

Institute of Infrastructure Technology Research and Management (IITRAM)

India vapor slugs were formed in PHP and gets randomly distributed in the pipe due to surface tension. The oscillation motion counteracts the pressure difference due to expansion and contraction of working fluid caused by absorption and dissipation of sensible heat. The operational mechanism of the PHP involves evaporation and condensation of thin liquid film, dynamic contact angle of the working fluid on tube wall, the growing and flowing of Taylor bubble and the coalescence of the small bubbles [7]. Therefore, although the structure of a PHP is very simple, the coupling of hydrodynamic and thermo- dynamic effects during the process of heat and mass transfer in the PHP make the operational mechanism of PHP very complicated and hard to be fully revealed $[8,9]$.

Extensive experimental and theoretical work have been conducted by many researchers over decades, studying complex characteristics of heat transfer and dynamic oscillating behavior inside PHP. In spite of its simple structure, the PHP working principles are very complex and yet a complete and validated design tool has not been developed [8].

For experimental research, various parameters such as geometric parameters of PHP, inner diameter, the crosssection shape of the PHP, channel configuration, the length of the evaporation and condensation section, the number of turns, etc. have been intensely studied. Various operational parameters such as charge ratio, heat flux, inclination angle, external force, etc. have also been researched [8].

For theoretical studies, many models describing dynamics inside CLPHP have been proposed. Wong et al. [10] used Lagrangian approach for theoretical modeling of PHPs in which they assumed the flow was under adiabatic conditions for the entire PHP. For the vapor plugs they have shown the pressure and velocity variations with time. Shafii and Faghri [11] used a U-shaped model of open PHP for the analysis of oscillating heat transfer. They developed conservation equations for each liquid and vapor slug by the control volume method. Their model was able to estimate slug flow direction and the ratio of sensible and latent heat. Mameli [12], considered mass, momentum and energy conservation using finite difference scheme. The results showed that heat transfer in both looped and unloopled PHPs is due mainly to the exchange of sensible heat. Givler and Martinez [13] took the homogeneous bubble model (HBM) for PHP simulation by FLOW-3D. They showed that most of the coalescing has taken place near the condenser, and liquid slugs agglomerated according to the direction of gravity. Lin [14] used commercial software fluent to consider the effects of surface tension and phase change on thermal performance of PHP. Nagwase [15] used star CCM Plus to model two phase flow in PHP with DI water as working fluid without considering temperature dependency of different parameters of working fluid. 
Though various numerical models have been proposed over last two decades, but only a few of them were able to obtain proper simulation of different PHPs. For example, Zuo et al. [16] gave one dimensional model for the thermal hydraulic simulation of a pulsating heat pipe and experimental validation was shown only for the thermal performance over filling ratio for a flat-plate PHP with embedded porous wick. Khandekar and Groll [17] suggested a lumped parameters model for the single loop of CLPHP. He assumed homogeneous fog flow which seems more suitable for thermosyphons than for the PHP. Hence overall still there is no effective numerical model to predict thermal performance of PHP.

To further explore the potential applications of PHPs technology, it is necessary to predict the thermal performance of CLPHPs by computational modeling and simulation. Hence in this work a computational model for CLPHP is developed to simulate the process of flow and heat transfer, including the oscillations and the temperature change due to evaporation and condensation occurring in bottom heating mode. Experimental results [18] were eventually used to verify the model established in this paper

\section{Computational Modelling of CLPHP}

\section{A. Physical Model}

A two dimensional physical model was developed using the commercial software package ANSYS FLUENT, to simulate the internal flow and heat transfer in a CLPHP. Water was used as working fluid. The copper was chosen as tube material. According to the experimental conditions [18] four turn PHP is divided into 3 parts; evaporator section, adiabatic section, and condenser section. The corresponding sections are shown in Fig 1. The geometric dimension of CLPHP are mentioned in Table 1.

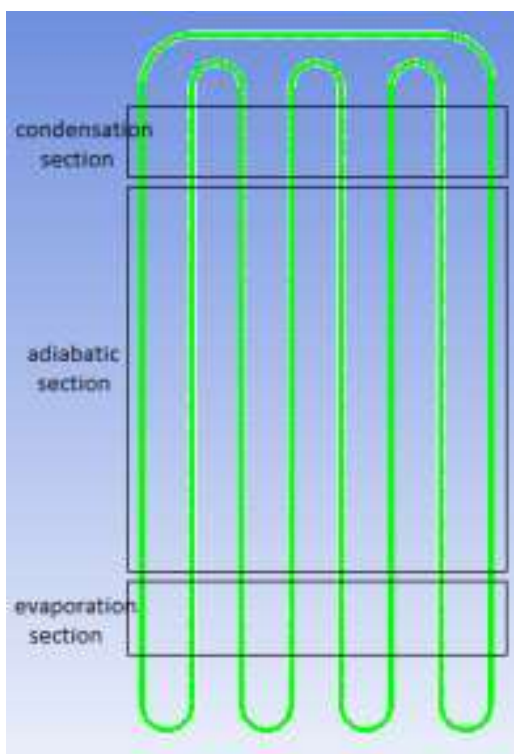

Fig 1. Geometric model
Table 1: Geometric dimension of physical model

\begin{tabular}{|c|c|}
\hline Parameter & Value $(\mathbf{m m})$ \\
\hline Inner diameter & 1.3 \\
\hline Outer diameter & 2.5 \\
\hline Evaporator length & 20 \\
\hline Condenser length & 20 \\
\hline Adiabatic length & 150 \\
\hline
\end{tabular}

The grid size was chosen according to Lin [14] with cell count equal to 8400 where minimum and maximum length of the element is taken equal to $0.026 \mathrm{~mm}$ and $2.6 \mathrm{~mm}$ respectively. Time step of $10^{-5}$ is chosen.

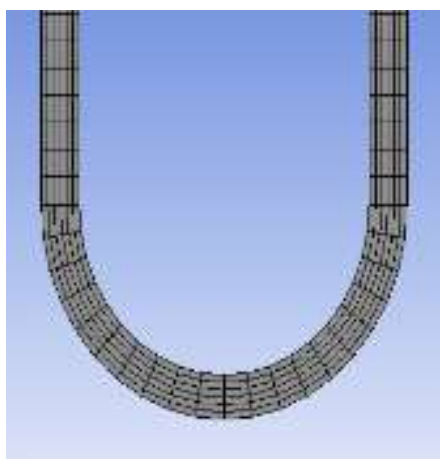

Fig 2. Mesh configuration of evaporator section

Figure 2 shows meshing configuration used in this study. Only a part of evaporator section has been shown in the figure for clear visualization of quadrilateral mesh used in the simulation.

\section{B. Computational Model Set-up}

Continuity equations, momentum equation and energy equations in VOF model were used to describe the motion of working fluid in a CLPHP [14]. Volume of fluid (VOF) model with two Eulerian phases, water and water-vapour with implicit body force formulation and $10^{-8}$ volume fraction cutoff was activated. Energy equation was turned on to account for heat transfer. Viscous model was selected laminar. Water vapor and water were taken as primary fluid and secondary fluid respectively. The density $(\rho)$ of water was taken to be temperature dependent and its value was calculated from steam table:

$$
\rho=80106.84-747.9185 \mathrm{~T}+2.35502 \mathrm{~T}^{2}-0.00247 \mathrm{~T}^{3}
$$

Gravitational acceleration was turned on and operating pressure of $4246.9 \mathrm{~Pa}$ was considered. As the CLPHP being a negative pressure system, it was assumed that the boiling temperature of water was $303 \mathrm{~K}$, according to the experimental observations. The phase change between liquid and vapor is carried out using evaporation-condensation model governed by Lee model [19]. The continuum surface force (CSF) model was used to consider the effect of surface tension. The value of surface tension was taken to 0.0712 $\mathrm{N} / \mathrm{m}$ along with wall adhesion. 
The heat flux in evaporation section depended on the power input. According to the general temperature requirement of electronic cooling and the experimental results [18], the heating powers are not over $100 \mathrm{~W}$. Hence maximum heat flux of $100 \mathrm{~W}$ is assigned at the evaporator. The convection heat transfer coefficient in condensation section was set to about $4000 \mathrm{~W} /\left(\mathrm{m}^{2} \mathrm{~K}\right)$. The adiabatic section was set as insulation boundary condition. The convection heat transfer coefficient for the other section which are in contact with air was set to $5 \mathrm{~W} / \mathrm{m}^{2} \mathrm{~K}$.

\section{Solution Methods}

The operating temperature was $301 \mathrm{~K}$ and the initialized filling ratio was taken as 50\% [18]. In the model solution the pressure velocity coupling was taken as PISO with skewness- neighbor coupling. Under discretization scheme, pressure interpolation was set to body force weighted; momentum, volume fraction and energy were taken as first order upwind. The relaxation factors for pressure, density and momentum were set to $0.3,1$, and 0.01 respectively. These control factors however were varied during the simulation to maintain the residuals in desired range. The flow characteristics and thermal resistance for CLPHP were obtained for time-step of $10^{-5} \mathrm{~s}$.

\section{Results and discussion}

The simulation was carried out for a time period of $13 \mathrm{~s}$ in order to obtain two phase flow process occurring in CLPHP and to determine its thermal resistance.

There was a temperature-rise period before the CLPHP developed into stable oscillation, which is clearly visible in

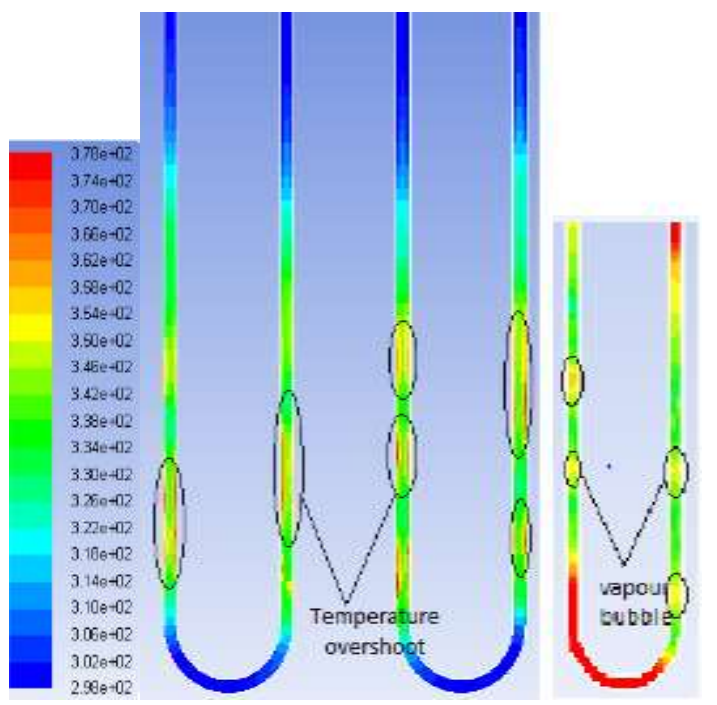

Fig. 3 Temperature overshoot resembling bubble growth at $1.5 \mathrm{~s}$

the Fig. 3. This operating temperature overshoot seen at $1.5 \mathrm{~s}$ of startup, represented the bubble generation in CLPHP, and corresponding vapour bubble generated is also observed. These observations clearly resembles startup performance of bubble generation in CLPHP.

Temperature plots of CLPHP with water as working fluid at different time can be seen in Fig. 4. Two phase flow process and pulsation of liquid plugs due to pressure difference between the evaporator and condenser was also
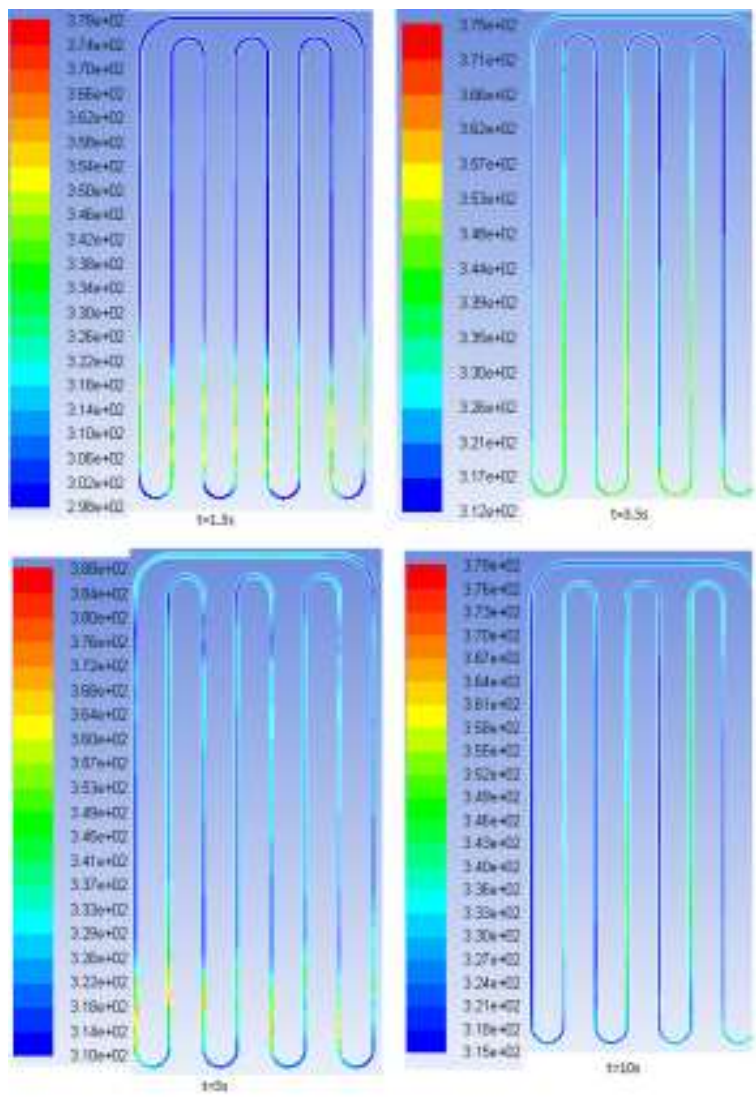

Fig. 4 Contours of temperature change in CLPHP operation process
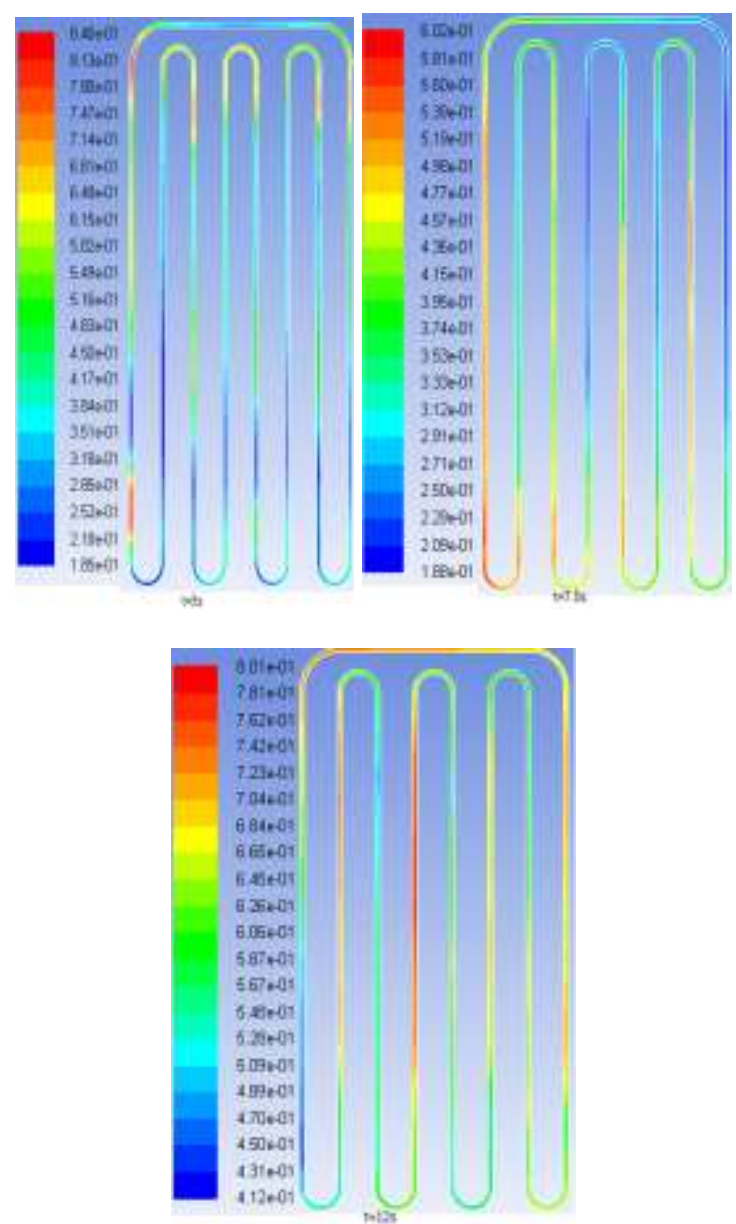

Fig. 5 Volume fraction of water at $5 \mathrm{~s}, 7.5 \mathrm{~s}$ and $12 \mathrm{~s}$. 
achieved as shown in the Fig. 5. It is found that pulsating behavior of liquid plugs in CLPHP in vertical bottom heated mode was attained after $7.5 \mathrm{~s}$ of startup.

The thermal resistance (R) of CLPHP is the ratio of temperature difference between the evaporator and condenser and corresponding heating power. Hence

$$
R=\frac{T_{e}-T_{c}}{Q}
$$

where $T_{e}$ is average temperature at evaporator; $T_{c}$ is average temperature of condenser, and $\mathrm{Q}$ is the heating power. Thermal resistance CLPHP with water as working fluid in vertical bottom heated mode was plotted against simulation time as shown in Fig. 6.

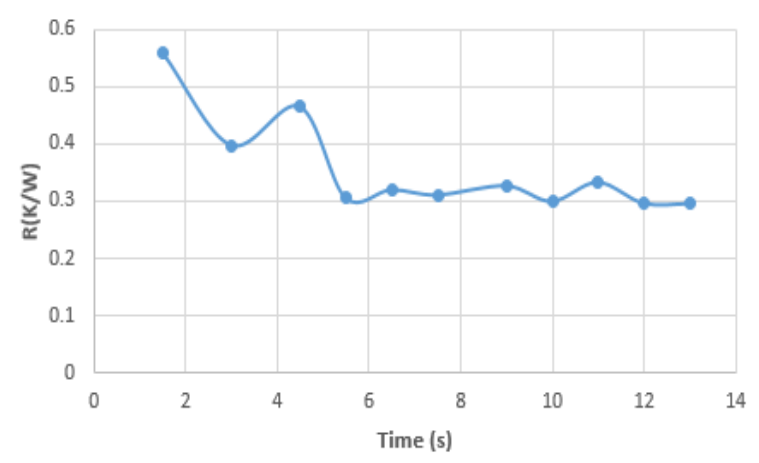

Fig 6. Graph of thermal resistance with time.

It can be seen from the figure that thermal resistance becomes almost constant after $7.5 \mathrm{~s}$ after which it can be treated as normal pipe with constant thermal conductivity. Hence it is also clear from the graph that steady pulsating flow is achieved at 7.5s. Also because of temperature overshoot at $1.5 \mathrm{~s}$ highest thermal resistance of $0.56 \mathrm{~K} / \mathrm{W}$ was achieved.

From the computational model developed lowest thermal resistance achieved was $0.295 \mathrm{~K} / \mathrm{W}$ whereas thermal resistance achieved in experimental work by Zirong Lin [15] was found to be $0.24 \mathrm{~K} / \mathrm{W}$. Hence the simulations results are in good agreement with respect to experimental work.

\section{Conclusions}

In this paper a computational model was developed to understand the heat transfer mechanism in CLPHP and to predict the thermal performance of CLPHP in vertical position with water as a working fluid. The effects of phase change using Lee model and surface tension using continuum surface model was considered. Following conclusion have been made from the study:

- It was shown that the simulation of CLPHP with unsteady model was able to predict the operation of CLPHP, including bubble formation and oscillation phenomena caused by the pressure difference.

- Overall the computational model shows good agreement with experimental work. Therefore, it can serve as a design tool for estimating the thermal performance of pulsating heat pipe with different geometries and working fluid, without the need of any experimental model.

\section{References}

[1] H.H. Yang, S. Khandekar, M. Groll, "Operational limit of closed loop pulsating heat pipes,” Appl. Therm. Eng., vol. 28, pp. 49-59, 2008.

[2] Akachi, H., Structure of a Heat Pipe, US patent, No. 4,921,041, 1990.

[3] Groll M, Khandekar S. Pulsating heat pipes: progress and prospects. In: Proceedings of international conference on energy and the environment, China; 2003.

[4] G. Burban, V. Ayel, A. Alexandre, Experimental investigation of a pulsating heat pipe for hybrid vehicle applications, Appl. Therm. Eng. 50 (1) (2013) 94-103.

[5] J. Clement, X. Wang, Experimental investigation of pulsating heat pipe performance with regard to fuel cell cooling application, Appl. Therm. Eng. 50 (1) (2013) 268-274.

[6] H. Yang, S. Khandekar, M. Groll, Performance characteristics of pulsating heat pipes as integral thermal spreaders, Int. J. Therm. Sci. 48 (4) (2009) 815-824.

[7] Khandekar S, Panigrahi PK, Lefèvre F, Bonjour J. Local hydrodynamics of flow in a pulsating heat pipe: a review. Front Heat Pipes 2010; (1):1-20.

[8] X. Han, X Wang, H Zheng. Review of the development of pulsating heat pipe for heat dissipation. Renewable and sustainable energy reviews, pp. 692-709.

[9] Zhang Y W, Faghri A. Advances and unsolved issues in pulsating heat pipes. Heat Transf Eng 2008; 29(1): 20-4

[10] Wong, T. N., Tong, B. Y., Lim, S. M., and Ooi, K. T., 1999, "Theoretical Modeling of Pulsating Heat Pipe," Proceedings of 11th International Heat Pipe Conference, Tokyo, Japan, pp. 159-163.

[11] Y.W. Zhang, A. Faghri, M.B. Shafii, Analysis of liquid-vapor pulsating flow in a U-shaped miniature tube, Int. J. Heat Mass Transf. 45 (2002) 2501-2508.

[12] A. Faghri, Y. Zhang, Thermal modeling of unlooped and looped pulsating heat pipes, J. Heat Transfer 123 (2001) 11591172 .

[13] R.C. Givler, M.J. Martinez, Modeling of Pulsating Heat Pipes, SANDIA REPORT, SAND2009-4520, Springer, August 2009.

[14] Z. Lin, S. Wang, R. Shirakashi, Simulation of a miniature oscillating heat pipe in bottom heating mode using CFD with unsteady modeling, Int. J. Heat Mass Transfer 57 (2) (2012) 642-656

[15] Nagwase S Y, Pachghare P R. Experimental and CFD analysis of closed loop pulsating heat pipe with DI-water. In: Proceedings of ICEETS, New York; 2013.

[16] Zuo, Z. J., North, M. T., and Ray L., Combined Pulsating and Capillary Heat Pipe Mechanism for Cooling of High Heat Flux Electronic, Internal Report, Thermacore, Inc., Lancaster, PA, USA, 2001

[17] Khandekar S., and Groll, M., Roadmap to Realistic Modeling of Closed Loop Pulsating Heat Pipes, Proc. 9th International Heat Pipe Symposium, Kuala Lumpur, Malaysia, November 2008.

[18] Z.R. Lin, S.F. Wang, J.J. Chen, et al., Experimental study on effective range of miniature oscillating heat pipes, Appl. Therm. Eng. 31 (5) (2011) 880-886.

[19] Fadhl B, Wrobel LC, Jouhara H (2015) CFD Modelling of a two-phase closed thermosyphon charged with R134a and R404a. Appl Therm Eng (78). pp.482-490 


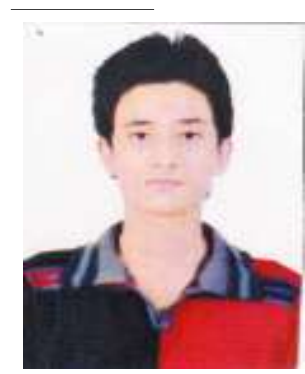

Ashutosh Gupta is B. Tech IIIrd year student in Mechanical Engineering Department at IITRAM Ahmedabad, Gujarat (India). His main research areas are in the field of heat transfer.

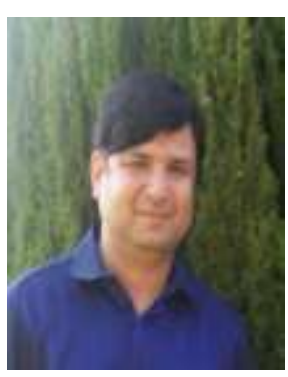

Ajit K Parwani is Assistant Professor in Mechanical Engineering Department at IITRAM Ahmedabad, Gujarat (India). He graduated from RGPV Bhopal and post graduated from DTU Delhi, and obtained his $\mathrm{PhD}$ from IIT Delhi (India), in 2013. He has been serving as an academician and researcher in technical institutes from 10 years in India. His main research areas are in the field of heat transfer, combustion and renewable energy. 